\title{
Recombinant adenovirus of SEA and CD80 genes driven by MMRE and mouse TERT promoter induce effective antitumor immune responses against different types of tumor cells in vitro and in vivo
}

\author{
SHAO-YAN SI ${ }^{1 *}, J_{U N-L I ~ L I U}{ }^{1 *}$, JUN-LIAN LIU ${ }^{2}$, BING-XIN XU ${ }^{1}$, \\ JIAN-ZHONG LI ${ }^{1}$, YA-YA QIN ${ }^{1}$ and SHU-JUN SONG ${ }^{1}$ \\ ${ }^{1}$ Center for Special Medicine and Experimental Research, ${ }^{2}$ Department of Dermatology, \\ 306th Hospital of PLA, Beijing 100101, P.R. China
}

Received September 12, 2016; Accepted February 6, 2017

DOI: $10.3892 /$ or.2017.5563

\begin{abstract}
Staphylococcus enterotoxin A (SEA) is a powerful immunostimulant and can stimulate $\mathrm{T}$ cells bearing certain $\mathrm{T}$-cell receptor $\beta$-chain variable regions when bound to major histocompatibility complex II molecules. SEA is widely used in research of antitumor therapy. The low affinity T-cell receptor (TCR) interaction with SEA in the absence of MHC class II antigens is sufficient for the induction of cytotoxicity but requires additional CD28/B7 signaling to result in proliferation of resting $\mathrm{T}$ cells. In this study, we constructed recombinant adenovirus (named as Ad-MMRE-mTERT-BIS) carrying membrane-expressing SEA (named as SEAtm) and CD80 driven by Myc-Max response elements (MMRE) and mouse telomerase reverse transcriptase (mTERT) promoter to reduce toxicity and to improve safety and efficiency. We demonstrated that Ad-MMRE-mTERT-BIS could make SEAtm and CD80 to co-express highly on the surface of Hepa1-6 and B16 cells, at low level on the surface of CT26 cells, but not in NIH3T3. Hepa1-6 and B16 cells infected by the recombinant adenovirus induced proliferation of $\mathrm{CD}^{+}$and $\mathrm{CD} 8^{+} \mathrm{T}$ cells and increased cytokine [interleukin (IL)-2, tumor necrosis factor (TNF)- $\alpha$, interferon (IFN)- $\gamma$ ] production in vitro. Intratumoral injection of Ad-MMRE-mTERT-BIS in hepatoma and melanoma mouse models induced tumor-specific cytotoxic $\mathrm{T}$ cells in the spleen. Moreover, hepatoma and melanoma xenografts were suppressed by treatment with Ad-MMRE-mTERT-BIS and
\end{abstract}

Correspondence to: Professor Shu-Jun Song, Center for Special Medicine and Experimental Research, 306th Hospital of PLA, 9 An Xiang Bei Li, Chao Yang, Beijing 100101, P.R. China

E-mail: shuj80@126.com

${ }^{*}$ Contributed equally

Key words: staphylococcus enterotoxin A, CD80, mTERT promoter, gene therapy the survival time of treated mice was prolonged. These findings suggest that recombinant adenovirus of SEA and CD80 genes driven by mTERT promoter could induce effective antitumor immune responses against different kinds of tumor cells in vitro and in vivo.

\section{Introduction}

Superantigens (SAgs) derived from bacterial or viral products are known as potent activators, which bind to both MHC class II molecules and specific $\mathrm{V} \beta$ regions of $\mathrm{T}$ cell receptors as an unprocessed protein, resulting in the activation of more than $10-25 \%$ of the T-cell population (1). The activation of lymphocytes by superantigen resulted in cytokine production, proliferation and cytotoxicity, and could elicit systemic antitumor immunity in vitro and in vivo (2-7). This property of SAgs has been used in cancer immunotherapy. Antitumor strategies of superantigen included antibody-targeted super antigen (8-13), superantigen-transmembrane sequence fusion protein (14), and gene therapy (15). Staphylococcus enterotoxin A (SEA) is a kind of superantigen and was widely used in research of antitumor therapy $(8-11,13,15)$. In our previous study (15), SEA was used for cancer gene therapy and induced efficient antitumor effects. The low affinity T-cell receptor (TCR) interaction of SEA in the absence of MHC class II antigens is sufficient for induction of cytotoxicity but requires additional CD28/B7 signaling to result in the proliferation of resting $\mathrm{T}$ cells (16). Previous studies have also shown that SEA, in combination with B7 costimulatory molecule, induced a stronger lymphocyte proliferation response and antitumor immunity than either SEA or B7 alone (17). In this study, SEA and B7 (CD80) genes were used to investigate their antitumor effects.

In the gene therapy of tumors, recombinant adenovirus is widely used for the transfer of foreign genes into tumor cells. However, the utilization of these vectors requires the specific and efficient expression of the transferred gene in tumor cells because infections of adenovirus to cells lack tissue specificity. One targeting strategy most often applied is the expression of 
the transgene controlled by a tumor-specific promoter, such as $\alpha$-fetoprotein (AFP) promoter. However, true tumor-specific promoter is rare, and often these promoters are useful only for the particular types of cancers. Previously, we constructed recombinant adenovirus carrying SEAtm gene driven by AFP enhancer/promoter, which could only be used for hepatocellular carcinoma. In vitro and in vivo studies have demonstrated that the human telomerase reverse transcriptase TERT (hTERT) promoter is highly active in $80-90 \%$ human cancer cells but not in normal differentiated human cells. Therefore, hTERT promoter has been widely used for targeted gene therapy to many types of cancers (18). Similarly, telomerase activities were at high levels in approximately $90 \%$ of mouse cancers or tumor-derived cell lines through TERT transcriptional upregulation (19).

In our previous study, we found the proximal 333-bp fragment was the core promoter of the mTERT gene in the cancer cells. The proximal 333-bp fragment of mTERT promoter was used to drive SEA and CD80 gene in our constructed recombinant adenovirus in order to be applied in various types of cancers. Myc family protein, groups of the helixloop-heilx/leucine zipper family, forms heterodimers with a partner protein, Max. This Myc-Max protein complex binds to the CACGTG sequence and activates transcription $(20,21)$. Myc and Max protein expression increased in many kinds of cancers. Myc-Max response elements (MMRE) were used to increase the hTERT promoter activity $(22,23)$.

In the present study, to extend the applicability of the gene therapy vectors for different kinds of tumors, we constructed a recombinant adenovirus carrying SEAtm and mouse CD80 gene driven by the proximal 333-bp fragment of mTERT promoter, upstream of which MMRE was used to increase the mTERT promoter activity. Then, antitumor effects of recombinant adenovirus were observed in hepatoma, colon cancer and melanoma in vitro and in vivo.

\section{Materials and methods}

Cell lines. Mouse hepatoma cell line Hepa1-6, melanoma cell line B16, colon cancer cell line CT26 and fibroblast cell line NIH3T3 were stored in our laboratory. All the cell lines were maintained in Roswell Park Memorial Institute (RPMI)-1640 (Gibco-BRL) or high glucose Dulbecco's modified Eagle's medium (DMEM), which were supplemented with $10 \%$ fetal bovine serum and penicillin/streptomycin in an atmosphere of $5 \% \mathrm{CO}_{2}$ chamber at $37^{\circ} \mathrm{C}$.

Antibodies. Phycoerythrin (PE)-conjugated anti-mouse CD80 (eBioscience, San Diego, CA, USA) and CD4 (eBioscience), APC-conjugated anti-mouse CD8 (eBioscience), rabbit anti-SEA (Abcam, Cambridge, MA, USA), fluorescein isothiocyanate (FITC)-conjugated donkey anti-rabbit IgG (Biolegend) and anti-mouse CD3 (eBioscience) were used in this study.

Recombinant adenovirus preparation. We used the AdEasy vector system (Qbiogene Inc.), that is, human adenovirus serotype 5 and rendered replication defective by the deletion of the E1 and E3 gene. Preparations of the recombinant adenoviruses were performed as previously described (25). Proliferation, purification and titering of adenovirus Ad(empty),
Ad-MMRE-mTERT-CD80 (carrying mouse CD80 gene only), Ad-MMRE-mTERT-SEAtm (carrying SEAtm gene only) or Ad-MMRE-mTERT-BIS (carrying mouse CD80 and SEAtm genes) were performed as described in the manufacturer's protocol. None of the stocks of virus used in the experiments contained detectable replication-competent viruses as evaluated by PCR assay, which used two pairs of primers to detect adenoviral E1A DNA.

Adenovirus-mediated SEA and CD80 expression in different cell lines in vitro. Hepa1-6, B16, CT26 or NIH3T3 cells were plated at a density of $5 \times 10^{5}$ cells/well in 6 -well culture plates $24 \mathrm{~h}$ before the adenoviruses infection. Immediately before the infection with Ad(empty), Ad-MMRE-mTERT-CD80, Ad-MMRE-mTERT-SEAtm or Ad-MMRE-mTERT-BIS, culture medium was aspirated and the adenoviruses were distributed over the cell monolayer at multiplicity of infection (MOI) of 100. The ratio of the number of adenovirus per cell was expressed as MOI. After $48 \mathrm{~h}$ of culture, the cells were digested with $0.02 \%$ ethylenediaminetetraacetic acid, washed in PBS containing 2\% calf serum, incubated with a rabbit anti-SEA polyclonal antibody and detected with FITC-labeled donkey anti-rabbit immunoglobulin $\mathrm{G}$ ( $\mathrm{IgG}$ ) and/or with PE-conjugated anti-mouse CD80. The analysis was performed by FCM.

The expression of SEA and CD80 on the surface of Hepa1-6 and B16 cells was also visualized by in situ immunofluorescent staining. Hepa1-6 or B16 cells were seeded at a density of $1 \times 10^{4}$ cells/well onto glass coverslips and grown for $48 \mathrm{~h}$ after infection with Ad-MMRE-mTERT-BIS at MOI 100. Cells were stained with the Abs at $4^{\circ} \mathrm{C}$ as described above and fixed with $4 \%$ paraformaldehyde in PBS for $30 \mathrm{~min}$, and then washed twice in PBS buffer. Finally, the coverslips were mounted onto glass slides. Fluorescence distribution was analyzed using a laser confocal scanning microscope.

Proportion of $\mathrm{CD}^{+}$and $\mathrm{CD} 8^{+} \mathrm{T}$ lymphocytes and cytokine production in splenocytes induced by tumor cells infected with the recombinant adenoviruses in vitro. Mouse splenocytes $\left(10^{7}\right.$ cells/well) were co-cultured with $10^{6}$ inactivated Hepa1-6 or B16 cells non-infected or infected with Ad(empty), Ad-MMRE-mTERT-CD80, Ad-MMRE-mTERT-SEAtm or Ad-MMRE-mTERT-BIS in 24-well plate and incubated for $48 \mathrm{~h}$ in a humidified chamber at $37^{\circ} \mathrm{C}, 5 \% \mathrm{CO}_{2} . \mathrm{CD}^{+}$and $\mathrm{CD}^{+} \mathrm{T}$ lymphocytes in splenocytes were stained with FITCconjugated anti-mouse CD3, PE-conjugated anti-mouse CD4 and APC-conjugated anti-mouse CD8 and analyzed by FCM. IL-2, TNF- $\alpha$, and IFN- $\gamma$ in cell culture supernatant were measured using ELISA kits (eBioscience).

Mouse tumor preparation. Female C57BL/6 mice, 6-8 weeks of age, were obtained from the Experimental Animal Center (Health Science Center, Peking University, Beijing, China) under strictly controlled specific-pathogen-free conditions. Principles of laboratory animal care (NIH publication no. 85-23, revised 1985) were followed, as well as the current version of the Chinese Law on the Protection of Animals. Mice were held in accordance with the permission of the responsible authority. Tumors were generated by subcutaneous injection of $10^{6}$ Hepa1-6 or B16 cells per mouse in $100 \mu \mathrm{l}$ of PBS on the 
Table I. Recombinant adenovirus Ad-MMRE-mTERT-BIS -mediated expression of SEA and CD80 on the tumor cells and NIH3T3.

\begin{tabular}{lccc}
\hline Cell lines & CD80-positive cells (\%) & SEAtm-positive cells (\%) & CD80- and SEA-positive cells (\%) \\
\hline NIH3T3 & $1.3 \pm 0.6$ & $0.9 \pm 0.3$ & $1.1 \pm 0.5$ \\
CT26 & $5.6 \pm 0.9$ & $5.7 \pm 1.1$ & $5.3 \pm 0.8$ \\
B16 & $41.2 \pm 1.9$ & $39.3 \pm 2.1$ & $39.1 \pm 1.5$ \\
Hepa1-6 & $89.7 \pm 2.9$ & $88.6 \pm 2.3$ & $87.2 \pm 3.1$ \\
\hline
\end{tabular}

right hind limb of C57BL/6 mice. Visible tumors developed at 7-9 days after tumor cell inoculation.

Tumor treatment in vivo. When the largest diameter of tumor exceeded $0.5 \mathrm{~cm}$, the Hepa1-6 hepatoma-bearing mice and B16 melanoma-bearing mice were, respectively, randomized into the following groups, and ten mice were included in each group: 1, B16-PBS (blank control); 2, B16-Ad(empty); 3, B16-CD80; 4, B16-SEAtm; 5, B16-BIS; 6, Hepa1-6-PBS (blank control); 7, Hepa1-6-Ad(empty); 8, Hepa1-6-CD80; 9, Hepa1-6-SEAtm; and 10, Hepa1-6-BIS. The mice in the control group were intratumorally injected with $100 \mu \mathrm{l}$ PBS per mouse. Mice in Ad(empty), CD80, SEAtm and BIS group were, respectively, injected intratumorally with $1 \times 10^{9} \mathrm{PFU}$ of Ad(empty), Ad-MMRE-mTERT-CD80, Ad-MMRE-mTERTSEAtm or Ad-MMRE-mTERT-BIS in $100 \mu \mathrm{l}$ PBS per mouse. The mice were injected twice a week for 2 weeks. Five mice in each group were sacrificed on day 14 after the last injection, and splenocytes were isolated for CTL activity and IFN- $\gamma$ producing cell assay. The other 5 mice in each group were monitored for survival ( $\leq 60$ days). Tumor sizes were measured before adenovirus injection and subsequently twice a week. The final tumor volume was measured on day 30 after tumor cell inoculation (before any deaths occurred) to ensure inclusion of the data from all the mice. Linear calipers were used to measure the longest diameter (a) and width (b). The tumor volume was calculated using the formula: $\left(a b^{2} / 2\right)$ and was plotted as the mean tumor volume of the group ( \pm standard error, SE) versus days post-tumor challenge.

Systemic antitumor cytotoxicity assays of CTLs in vivo. The CytoTox 96 non-radioactive cytotoxicity assay (Promega, Madison, WI, USA) was performed to measure the cytotoxic activity of the splenocytes in mice bearing tumors injected with PBS, Ad(empty), Ad-MMRE-mTERT-CD80, Ad-MMRE-mTERT-SEAtm or Ad-MMRE-mTERT-BIS, according to the manufacturer's protocol. Briefly, splenocytes of mice in each group were prepared 14 days after the last injection. Hepa1-6 or B16 cells in RPMI-1640 medium with $10 \%$ FBS were used as the targets for the CTL assays. Targets $\left(2 \times 10^{5}\right.$ cells/well) were mixed with splenocytes at effector: target (E:T) ratios of 50:1, 25:1, and 12.5:1, and incubated for $4 \mathrm{~h}$ in a humidified incubator at $37^{\circ} \mathrm{C}, 5 \% \mathrm{CO}_{2}$. Lysis solution was added to a portion of the target cells, prior to centrifugation, as a maximum LDH release control. Supernatant $(50 \mu 1)$ was transferred to the enzymatic assay plate after centrifugation, $50 \mu \mathrm{l}$ of the substrate mix was added to each well; the plate was covered to protect it from light, and incubated for $30 \mathrm{~min}$ at room temperature. Stop solution $(50 \mu 1)$ was added to each well and the absorbance was recorded at $490 \mathrm{~nm}$. The percentage of specific lysis was determined according to the following formula: [A (experimental)-A (effector spontaneous)-A (target spontaneous)x100)/[A (target maximum)-A (target spontaneous)].

IFN- $\gamma$ producing cell frequencies in vivo. Mouse IFN- $\gamma$ ELISpot assay was performed in PVDF bottomed 96-well plates by using a murine IFN- $\gamma$ ELISpot kit (Millipore, Bedford, MA, USA) according to the manufacturer's instructions. Briefly, plates were coated overnight at $4^{\circ} \mathrm{C}$ with anti-IFN- $\gamma$ capture antibody and washed three times with PBST (PBS+0.05\% Tween-20). Plates were blocked for $2 \mathrm{~h}$ with $2 \%$ skimmed dry milk/PBS. Splenocytes $\left(1 \times 10^{6}\right.$ cells/well $)$ of mice after treatment were then co-cultured with the MMC inactivated Hepa1- 6 cells or B16 (5x10 /well) and incubated for $24 \mathrm{~h}$ at $37^{\circ} \mathrm{C}$. Only splenocytes were added into wells as negative control. Cells were then removed and a biotinylated IFN $-\gamma$ detection antibody was added and incubated for $1 \mathrm{~h}$. Following extensive washing with PBST and PBS, the plates were incubated with streptavidin-alkaline phosphatase for $1 \mathrm{~h}$ at $37^{\circ} \mathrm{C}$. Spots were visualized by the addition of the alkaline phosphatase substrate BCIP/NBT. The number of dots in each well was counted by two separate investigators in a blinded manner using a dissecting microscope.

Statistical analysis. One-way analysis of variance (ANOVA) was performed to determine differences of immune response among the various treatment groups. Newman-Keuls tests were performed as post-hoc analysis for one-way ANOVA. The antitumor effects were considered statistically significant when the $\mathrm{P}$-value was $<0.05$.

\section{Results}

Recombinant adenovirus-mediated expression of SEA and CD80 on the tumor cells. Hepa1-6, B16, CT26 and NIH3T3 cells were infected with the recombinant adenovirus Ad-MMRE-mTERT-BIS. Flow cytometric analysis showed that SEAtm and CD80 proteins were efficiently co-expressed on the surface of the infected Hepa1-6 and B16 cells, the expression of SEAtm and CD80 proteins in Hepa1-6 was stronger than that in B16. A few infected CT26 cells and none of infected NIH3T3 cells expressed SEAtm and CD80 proteins (Table I). There were no significant differences between SEAtm and CD80 expression among the infected Hepa1-6 cells or B16 cells. To determine the distribution of CD80 and SEAtm protein on the infected cells, the cell images were visualized by laser confocal microscopy (Fig. 1). 
1

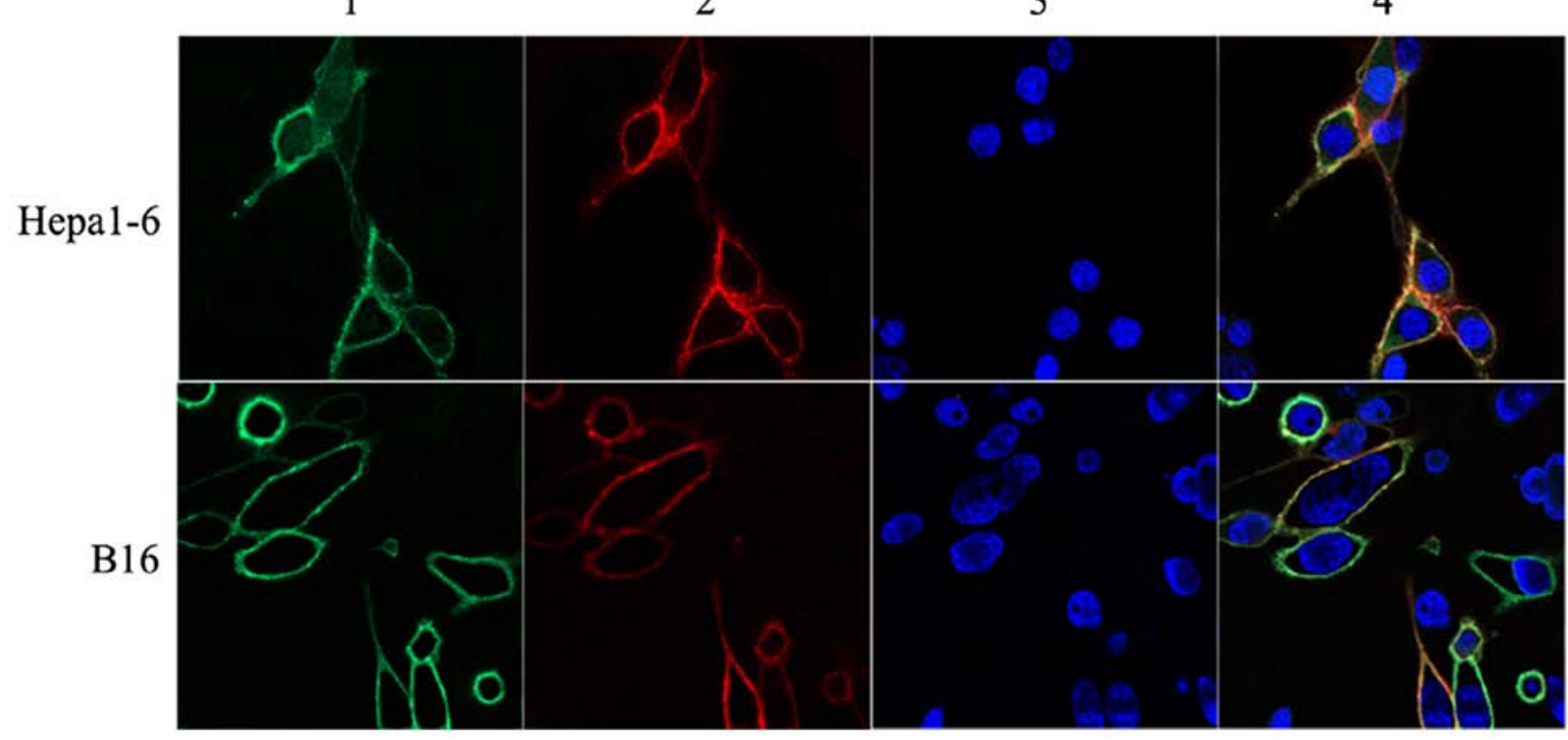

Figure 1. Laser confocal microscopic analysis of the Hepa1-6 and B16 cells infected with the recombinant adenovirus Ad-MMRE-mTERT-BIS. (1) The SEA protein expression (FITC labeled) is presented in green. (2) CD80 protein expression (PE labeled) is presented in red. (3) Nucleus (DAPI staining) is presented in blue. (4) Membrane of Hepa1-6 and B16 cells co-expressing SEA and CD80 in the merged image is presented in yellow.

Since a few infected CT26 cells expressed CD80 and SEAtm protein, we only observed CD80 and SEAtm protein expression on the surface of B16 and Hepa1-6 cells under laser confocal microscopy.

Tymphocyte sub-population proliferation induced by tumor cells infected the recombinant adenoviruses in vitro. The biological activity of CD80 and/or SEA expressed on the surface of Hepa1-6 and B16 cells in vitro was determined by the influence on the proportion of lymphocyte sub-population. The results are shown in Table II. The numbers of $\mathrm{CD}^{+}, \mathrm{CD}^{+}$ $\mathrm{CD}^{+}$, and $\mathrm{CD}^{+} \mathrm{CD}^{+} \mathrm{T}$ lymphocytes in cultures induced by tumor cells infected with Ad-MMRE-mTERT-BIS, Ad-MMRE-mTERT-CD80 or Ad-MMRE-mTERT-SEAtm increased as compared with those induced by non-infected tumor cells or tumor cells infected with Ad(empty) $(\mathrm{P}<0.05)$. The numbers of $\mathrm{CD}^{+}$and $\mathrm{CD}^{+}{ }^{+} \mathrm{CD} 4^{+} \mathrm{T}$ lymphocytes induced by tumor cells infected with Ad-MMRE-mTERT-BIS were the highest among all groups $(\mathrm{P}<0.05)$. The numbers of $\mathrm{CD}^{+}$and $\mathrm{CD}^{+}{ }^{+} \mathrm{CD} 4{ }^{+} \mathrm{T}$ lymphocytes in cultures induced by tumor cells infected with Ad-MMRE-mTERT-BIS or Ad-MMRE-mTERT-SEAtm increased compared to that in cultures induced by tumor cells infected with Ad-MMREmTERT-CD80 $(\mathrm{P}<0.05)$. There were no statistical differences in the number of $\mathrm{CD}^{+} \mathrm{CD}^{+} \mathrm{T}$ lymphocytes among cultures with tumor cells infected with Ad-MMRE-mTERT-BIS, Ad-MMRE-mTERT-SEAtm and Ad-MMRE-mTERT-CD80 $(\mathrm{P}>0.05)$.

Cytokine production of splenocytes induced by tumor cells infected by the recombinant adenoviruses in vitro. Splenocytes were isolated from a mouse and stimulated with inactivated Hepa1-6 or B16 cells non-infected or infected with Ad(empty), Ad-MMRE-mTERT-CD80, Ad-MMRE-mTERTSEAtm or Ad-MMRE-mTERT-BIS and cultured for $48 \mathrm{~h}$. The cytokines IL- 2, IFN- $\gamma$ and TNF- $\alpha$ in cell culture supernatant were detected. The results are shown in Table III. The levels of the three cytokines in splenocytes induced by the tumor cells infected with Ad-MMRE-mTERT-BIS were the highest in all the groups. The levels of the three cytokines in splenocytes induced by tumor cells infected with Ad-MMRE-mTERTSEAtm, the levels of the three cytokines in splenocytes induced by Hepa1- 6 cells or IL- 2 and TNF- $\alpha$ in splenocytes induced by $\mathrm{B} 16$ cells after infection with Ad-MMRE-mTERT-CD80 increased as compared with those induced by non-infected tumor cells or infected with Ad(empty). The levels of IFN- $\gamma$ induced by Hepa1- 6 cells and TNF- $\alpha$ induced by B16 cells infected with Ad-MMRE-mTERT-SEAtm were higher than those induced by the same cells infected with Ad-MMREmTERT-CD80.

IFN- $\gamma$-producing cell frequencies of splenic lymphocytes from the mice. Spleen lymphocytes were isolated from the mice (in triplicate) 14 days after the last injection, co-cultured with inactivated Hepa1-6 or B16 cells (treated with MMC, $100 \mu \mathrm{g} / \mathrm{ml}$ at $37^{\circ} \mathrm{C}$ for $1 \mathrm{~h}$ ) for $24 \mathrm{~h}$. Cells were removed and IFN- $\gamma$-producing cell frequency was determined for each group of mice with different treatments. As shown in Fig. 2, IFN- $\gamma$-producing cell frequencies of lymphocytes in Hepa1-6 hepatoma-bearing mice or B16 myeloma-bearing mice injected with Ad-MMRE-mTERT-BIS was the highest among all groups. The IFN- $\gamma$-producing cell frequencies in the mice injected with Ad-MMRE-mTERT-CD80 and Ad-MMRE-mTERT-SEAtm were much higher than those in mice injected with Ad(empty) and PBS $(\mathrm{P}<0.05)$.

CTL activity of splenocytes of the mice. Splenocytes isolated from mice in different groups 14 days after the last injection and used as CTL effector cells, and tested against Hepa1-6 or B16 cells as target cells, CTL activities were determined at 
Table II. The proportion of T lymphocyte sub-populations in splenocytes co-cultured with tumor cells infected with the recombinant adenoviruses in vitro.

\begin{tabular}{|c|c|c|c|c|c|c|}
\hline \multirow[b]{2}{*}{ Group } & \multicolumn{2}{|c|}{$\mathrm{CD}^{+} \mathrm{T}$ cells $(\%)$} & \multicolumn{2}{|c|}{$\mathrm{CD}^{+}{ }^{+} \mathrm{CD} 4^{+} \mathrm{T}$ cells $(\%)$} & \multicolumn{2}{|c|}{$\mathrm{CD}^{+} \mathrm{CD}^{+} \mathrm{T}$ cells $(\%)$} \\
\hline & Hepa1-6 & B16 & Hepa1-6 & B16 & Hepa1-6 & B16 \\
\hline Non-infection & $77.87 \pm 0.88$ & $67.87 \pm 0.78$ & $52.79 \pm 0.47$ & $45.34 \pm 0.57$ & $26.36 \pm 0.53$ & $21.36 \pm 0.59$ \\
\hline Ad(empty) & $78.28 \pm 0.91$ & $67.74 \pm 0.82$ & $52.26 \pm 0.91$ & $45.58 \pm 0.95$ & $26.02 \pm 0.08$ & $22.16 \pm 0.78$ \\
\hline Ad-MMRE-mTERT-CD80 & $87.13 \pm 0.90^{\mathrm{a}, \mathrm{b}}$ & $78.00 \pm 0.79^{\mathrm{a}, \mathrm{b}}$ & $53.27 \pm 0.93$ & $46.23 \pm 0.85$ & $33.86 \pm 1.72^{\mathrm{a}, \mathrm{b}}$ & $31.77 \pm 0.79^{\mathrm{a}, \mathrm{b}}$ \\
\hline Ad-MMRE-mTERT-SEAtm & $90.41 \pm 1.20^{\mathrm{a}, \mathrm{b}, \mathrm{c}}$ & $87.93 \pm 0.95^{\mathrm{a}, \mathrm{b}, \mathrm{c}}$ & $57.27 \pm 0.77^{\mathrm{a}, \mathrm{b}, \mathrm{c}}$ & $57.17 \pm 0.97^{\mathrm{a}, \mathrm{b}, \mathrm{c}}$ & $33.14 \pm 1.14^{\mathrm{a}, \mathrm{b}}$ & $30.76 \pm 0.96^{\mathrm{a}, \mathrm{b}}$ \\
\hline Ad-MMRE-mTERT-BIS & $94.53 \pm 0.50^{\mathrm{a}, \mathrm{b}, \mathrm{c}, \mathrm{d}}$ & $91.75 \pm 0.92^{\mathrm{a}, \mathrm{b}, \mathrm{c}, \mathrm{d}}$ & $61.86 \pm 0.99^{\mathrm{a}, \mathrm{b}, \mathrm{c}, \mathrm{d}}$ & $60.46 \pm 0.89^{\mathrm{a}, \mathrm{b}, \mathrm{c}, \mathrm{d}}$ & $32.67 \pm 1.48^{\mathrm{a}, \mathrm{b}}$ & $31.29 \pm 1.08^{\mathrm{a}, \mathrm{b}}$ \\
\hline
\end{tabular}

${ }^{a, b, c, d} \mathrm{P}<0.05$ as compared with non-infected tumor cells, tumor cells infected Ad(empty), Ad-MMRE-mTERT-CD80 or Ad-MMRE-mTERTSEAtm, respectively. The numerical values were the mean \pm SD in each group (in triplicate).

Table III. Cytokine production of splenocytes induced by tumor cells infected by the recombinant adenoviruses in vitro.

\begin{tabular}{|c|c|c|c|c|c|c|}
\hline \multirow[b]{2}{*}{ Group } & \multicolumn{2}{|c|}{$\mathrm{IL}-2$ (pg/ml) } & \multicolumn{2}{|c|}{$\mathrm{IFN}-\gamma(\mathrm{pg} / \mathrm{ml})$} & \multicolumn{2}{|c|}{$\mathrm{TNF}-\alpha(\mathrm{pg} / \mathrm{ml})$} \\
\hline & Hepa1-6 & B16 & Hepa1-6 & B16 & Hepa1-6 & B16 \\
\hline Non-infection & $7.9 \pm 0.7$ & $7.3 \pm 1.1$ & $37.6 \pm 6.7$ & $59.7 \pm 11.6$ & $28.3 \pm 1.5$ & $35.9 \pm 0.9$ \\
\hline Ad(empty) & $8.2 \pm 1.5$ & $7.3 \pm 0.7$ & $34.6 \pm 13.9$ & $60.8 \pm 7.1$ & $28.2 \pm 2.5$ & $35.6 \pm 1.8$ \\
\hline Ad-MMRE-mTERT-CD80 & $22.6 \pm 4.2^{\mathrm{a}, \mathrm{b}}$ & $12.6 \pm 1.0^{\mathrm{a}, \mathrm{b}}$ & $206.5 \pm 51.8^{\mathrm{a}, \mathrm{b}}$ & $65.8 \pm 12.9$ & $83.5 \pm 5.9^{\mathrm{a}, \mathrm{b}}$ & $69.9 \pm 3.5^{\mathrm{a}, \mathrm{b}}$ \\
\hline Ad-MMRE-mTERT-SEAtm & $19.8 \pm 6.7^{\mathrm{a}, \mathrm{b}}$ & $13.3 \pm 0.9^{\mathrm{a}, \mathrm{b}}$ & $352.4 \pm 26.7^{\mathrm{a}, \mathrm{b}, \mathrm{c}}$ & $74.8 \pm 7.0^{\mathrm{a}, \mathrm{b}}$ & $91.6 \pm 6.6^{\mathrm{a}, \mathrm{b}}$ & $89.1 \pm 6.8^{\mathrm{a}, \mathrm{b}, \mathrm{c}}$ \\
\hline Ad-MMRE-mTERT-BIS & $162.6 \pm 5.9^{\mathrm{a}, \mathrm{b}, \mathrm{c}, \mathrm{d}}$ & $70.7 \pm 3.9^{\mathrm{a}, \mathrm{b}, \mathrm{c}, \mathrm{d}}$ & $444.0 \pm 36.2^{\mathrm{a}, \mathrm{b}, \mathrm{c}, \mathrm{d}}$ & $349.3 \pm 24.5^{\mathrm{a}, \mathrm{b}, \mathrm{c}, \mathrm{d}}$ & $107.4 \pm 15.6^{\mathrm{a}, \mathrm{b}, \mathrm{c}, \mathrm{d}}$ & $159.0 \pm 7.0^{\mathrm{a}, \mathrm{b}, \mathrm{c}, \mathrm{d}}$ \\
\hline
\end{tabular}

a,b,c,dP $<0.05$ as compared with non-infected tumor cells, tumor cells infected Ad(empty), Ad-MMRE-mTERT-CD80 or Ad-MMRE-mTERTSEAtm, respectively. The concentrations of cytokines were the mean \pm SD in each group (in triplicate).

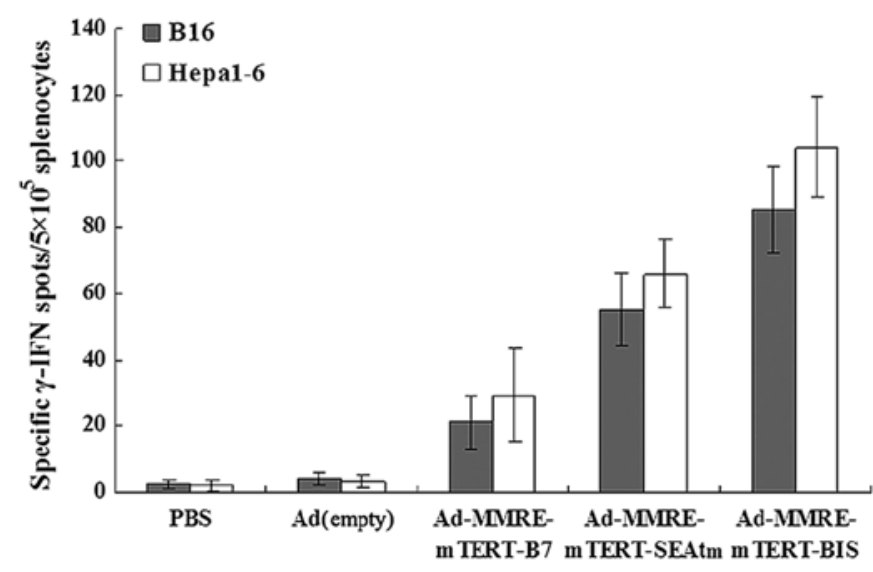

Figure 2. IFN- $\gamma$-producing cell frequency in the tumor-bearing mice treated with different vaccines was determined by an ELISpot assay. Splenocytes were isolated from the treated mice 14 days after the last injection. The splenocytes were co-cultured with inactivated tumor cells for $24 \mathrm{~h}$. The number of IFN- $\gamma$-producing tumor-specific $\mathrm{T}$ cell precursors was determined by using the ELISpot assay. The spot numbers are the means \pm SE in each group.

effector:target (E:T) ratios of 12.5:1, 25:1, and 50:1 by a standard CytoTox 96 non-radioactive cytotoxicity assay. As shown in Fig. 3A and B, lymphocytes derived from the mice treated with the Ad-MMRE-mTERT-BIS showed the highest CTL activities in all the groups. The CTL activities of the mice treated with Ad-MMRE-mTERT-CD80 and Ad-MMREmTERT-SEAtm were much higher than those of mice treated with Ad(empty) and PBS (P<0.05).

Antitumor effects of the recombinant adenoviruses in vivo. The results in Fig. 4 showed that tumor growth in mice treated with recombinant adenoviruses Ad-MMRE-mTERT-CD80, Ad-MMRE-mTERT-SEA or Ad-MMRE-mTERT-BIS was markedly inhibited as compared with that in mice treated with PBS or Ad(empty) from day 16 in Hepa1-6 tumorbearing mice (Fig. 4A) and from day 19 in B16 tumor-bearing mice (Fig. 4B; $\mathrm{P}<0.05$ ), the inhibition of the dual-gene therapy was significantly stronger than that of single gene therapy $(\mathrm{P}<0.05)$, there were no significant differences in tumor growth inhibition between the groups treated with Ad-MMRE-mTERT-CD80 and Ad-MMRE-mTERT-SEA $(\mathrm{P}>0.05)$. Five tumor-bearing mice in each group were monitored for their survival period. The results in Fig. 5 show that Hepa1-6 (Fig. 5A) or B16 (Fig. 5B) tumor-bearing mice treated with Ad-MMRE-mTERT-CD80, Ad-MMRE-mTERT-SEA or Ad-MMRE-mTERT-BIS survived longer than mice treated with $\mathrm{PBS}$ and Ad(empty) $(\mathrm{P}<0.05)$, tumor-bearing mice treated with Ad-MMRE-mTERT-BIS survived the longest of all the groups, there was no significant difference in the survival period between the mice treated with Ad-MMREmTERT-CD80 or Ad-MMRE-mTERT-SEA (P>0.05), two 

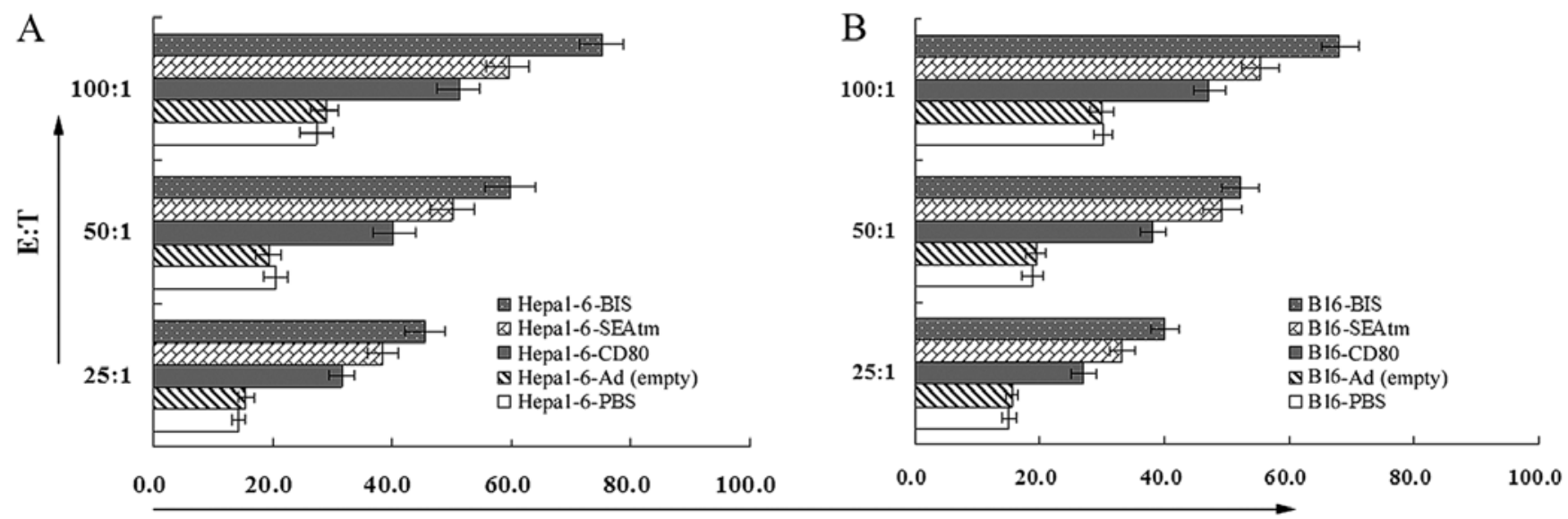

CTLs activity (\%)

Figure 3. CTL cell activity in Hepa1-6 hepatoma (A) and B16 melanoma (B) -bearing mice treated with different adenovirus was determined by a standard CytoTox 96 non-radioactive cytotoxicity assay. Data are presented as mean specific lysis of triplicate values \pm standard deviation. Splenocytes were isolated from the treated mice 14 days after the last injection and used as CTL effector cells. The Hepa1-6 and B16 cells were used as target cells. The CTL activity was determined at effector:target (E:T) ratios of 12.5:1, 25:1, and 50:1.
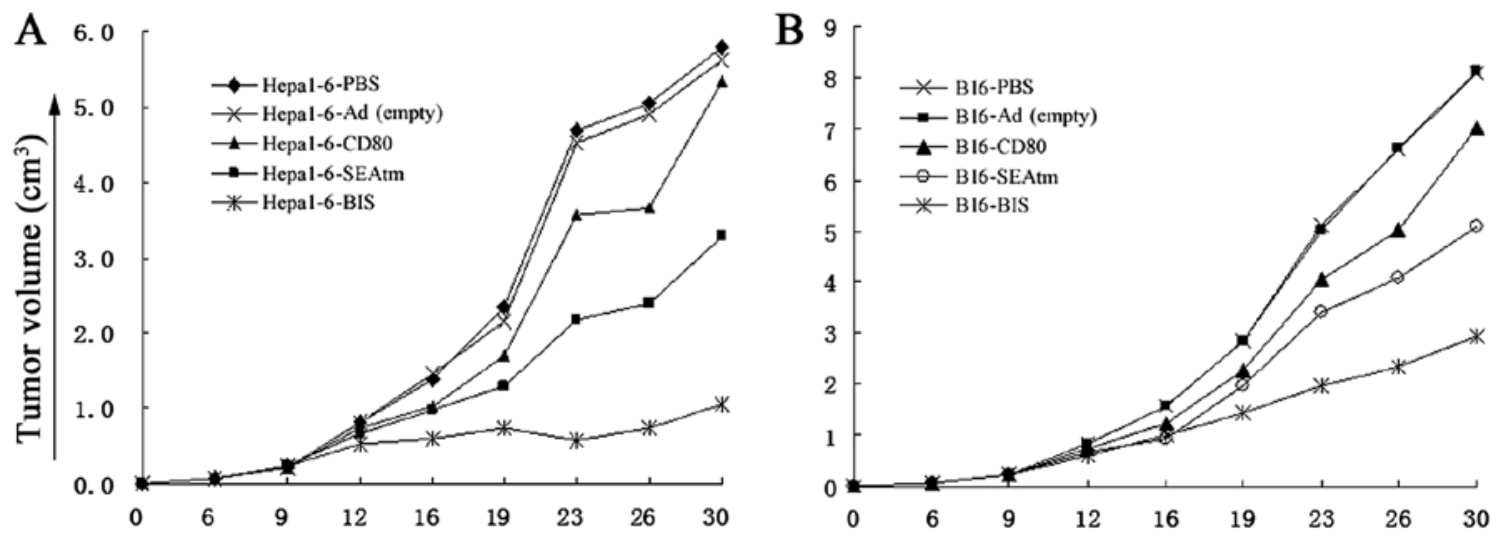

Days after tumor cells challenge

Figure 4. Ad-MMRE-mTERT-BIS decrease tumor growth of tumor-bearing mice. Mice subcutaneously inoculated with 1x10 ${ }^{6}$ Hepa1-6 (A) or B16 (B) tumor cells were intratumorally injected with PBS, Ad(empty), Ad-MMRE-mTERT-CD80, Ad-MMRE-mTERT-SEA or Ad-MMRE-mTERT-BIS. Tumor sizes are expressed as the mean diameter of the longest $\mathrm{x}$ the shortest diameter ${ }^{2} / 2$ as measured by a digital caliper twice a week for 30 days after tumor cell inoculation. Tumor growth was significantly decreased in mice treated with Ad-MMRE-mTERT-BIS compared to PBS, Ad(empty), Ad-MMRE-mTERT-CD80 or Ad-MMRE-mTERT-SEA from day $16, \mathrm{P}<0.05$.

mice in Hepa1-6-BIS group and one mouse in B16-BIS group had a survival period exceeding 60 days.

\section{Discussion}

As a SAg, SEA is a powerful immunostimulant. Previous studies have demonstrated that SEA anchoring onto MHC-IInegative tumor cells through antibodies directs T cell-mediated cytotoxicity against these tumors with reduced toxicity against normal MHC-II ${ }^{+}$cells (24-27). In addition, genetically engineered fusion protein of SEA with the transmembrane region sequence of c-erb-B2 could anchor on the surface of tumor and was capable of eliciting systemic antitumor immunity without any measured toxicity (28). These results indicated that the SAg anchoring on MHC-II-negative tumor cells assumes $\mathrm{T}$ cell stimulation, but it circumvents conventionally defined MHC 'presentation'. Furthermore, the anchored SAg showed a greater reduction in MHC class II binding compared to native forms and could elicit MHC-II-independent T cell stimulation in vitro as long as co-stimulatory signals were provided $(29,30)$. To decrease systemic activation as a consequence of SAg-MHC class II interaction with monocytes and B cells, and to localize the cytotoxic capabilities of SAg-activated T cells to tumor sites, the membrane-expressing SEA (31) was used in our study. To efficiently stimulate T cells, CD80 was simultaneously transduced into tumor cells. CD80 binds CD28 of T cells and provides the second signal for activation of $\mathrm{T}$ cells.

Pericuesta et al (32) generated the construct mTERT-GFP using the mTER gene promoter of 1,2 or $5 \mathrm{~kb}$ upstream of the first ATG of the open reading frame of mTERT gene to promote the expression of EGFP. In their transgenic model, no fluorescent expression of the mTERT-EGFP construct could be identified in adult tissues. This suggests that although telomerase activity exists in colon, liver, ovary, and testis, there 


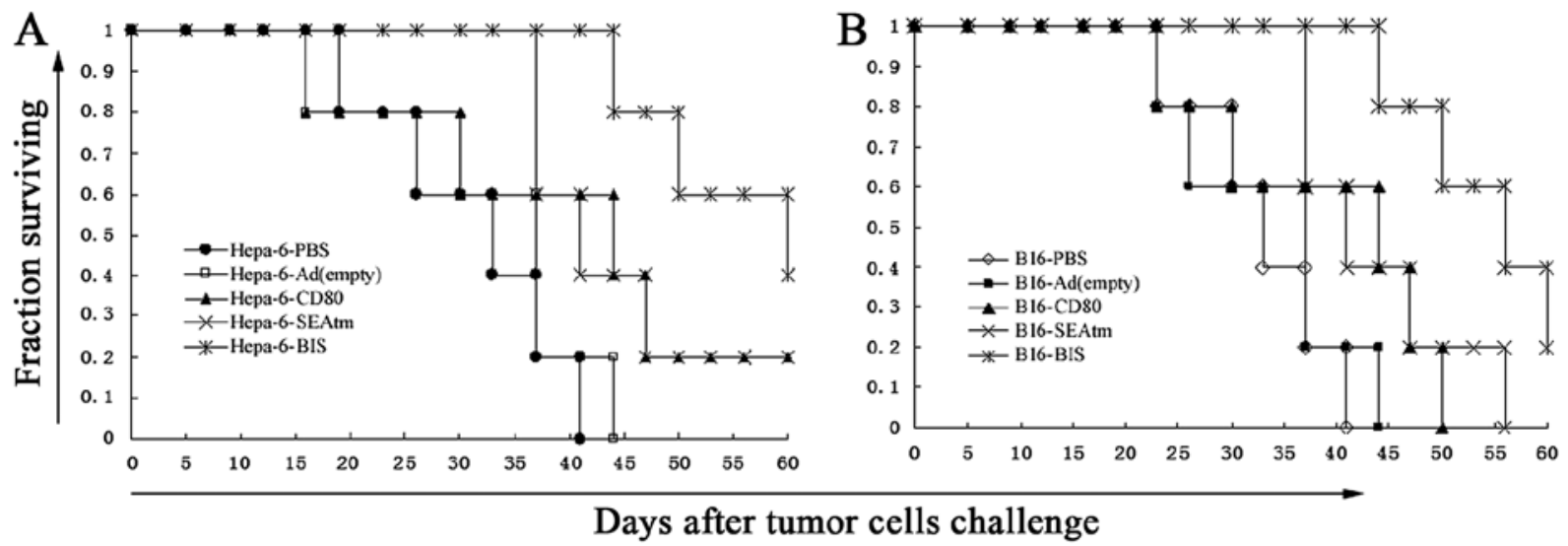

Figure 5. Ad-MMRE-mTERT-BIS enhance the survival of tumor-bearing mice. Mice subcutaneously inoculated with 1x10 ${ }^{6}$ Hepa1-6 (A) or B16 (B) tumor cells were treated with PBS, Ad(empty), Ad-MMRE-mTERT-CD80, Ad-MMRE-mTERT-SEA or Ad-MMRE-mTERT-BIS. Five mice in each group were observed for survival duration (for $\leq 60$ days). Survival of tumor-bearing mice was significantly enhanced by the treatment with Ad-MMRE-mTERT-BIS compared to that of mice treated with PBS, Ad(empty), Ad-MMRE-mTERT-CD80 or Ad-MMRE-mTERT-SEA, P<0.05.

is a tight repression system of mTERT gene promoter in adult mouse tissues in physiological conditions. In our previous study (33), we found the proximal 333-bp fragment was the core promoter of the mTERT gene in the cancer cells. The proximal 333-bp fragment was able to make SEA express on the surface of hepatoma cell line Hepa1-6, melanoma cell line B16, colon cancer cell line CT26, but not in the fibroblast NIH3T3 cells. In our study, SEAtm and CD80 gene driven by mTERT promoter in the recombinant adenovirus were constructed. The results showed that SEAtm and CD80 were co-expressed in $87.2 \%$ of Hepa1-6 cells, $39.1 \%$ of B16 cells and 5.3\% of CT26 cells, but not in fibroblast cell line NIH3T3 cells after infection with the recombinant adenovirus Ad-MMRE-mTERT-BIS. The positive rates of SEA and CD80 in Hepa1-6 cells, B16 cells and CT26 cells were different. The possible reasons are that the infection efficiency and expression efficiency of the same recombinant adenovirus Ad-MMRE-mTERT-BIS are different in different tumor cells. Ad-MMRE-mTERT-BIS was not used to treat CT26 colon cancer because the positive rate of SEA and CD80 in CT26 cells was very low after infection with Ad-MMRE-mTERT-BIS.

SEA are known as potent activators of $\mathrm{T}$ lymphocytes and effcient inducers of cytokine production $(34,35)$. Various cytokines, such as interleukin, interferon and tumor necrosis factor, can destroy vascular endothelial cells of the tumors, promote thrombosis and reduce blood supply to the tumor tissues, resulting in tumor cell necrosis and apoptosis. Cytokines also stimulate proliferation and differentiation of $\mathrm{T}$ cells, which will in return produce more cytokines, so as to form endogenously circulating biological effects and speed up the apoptosis of cancer cells. Cytokines can suppress tumor growth both directly and synergistically (35). In addition, cytokines can induce LAK activity, activate natural killer cells and macrophages $(10,36,37)$, facilitate penetration of high molecular weight proteins and upregulate cell adhesion molecules and MHC molecule expression on tumor cells. In our results, SEA expressed on the surface of Hepa1-6 and B16 cells was biologically active in vitro, as shown by the ability of Hepa1-6 and B16 cells infected by Ad-MMREmTERT-BIS to elicit proliferation and cytokine production of splenocytes. After stimulation with Hepa1-6 and B16 infected by Ad-MMRE-mTERT-BIS, Ad-MMRE-mTERT-SEAtm or Ad-MMRE-mTERT-CD80, the proportion of $\mathrm{CD}^{+}, \mathrm{CD}^{+}$ or/and $\mathrm{CD}^{+} \mathrm{T}$ cells in splenocytes and the level of IL-2, IFN- $\gamma$ and TNF- $\alpha$ produced by splenocytes increased. SEA and CD80 co-expressed tumor cells could more effectively activate splenocytes than SEA or CD80 expressed tumor cells.

Endogenously produced IFN- $\gamma$ protects the host from not only growing transplanted tumors, but also the formation of primary chemically-induced and spontaneous tumors (38-42) and plays a crucial role for the eradication of tumors in vivo (43). Injection of neutralizing mAbs for IFN- $\gamma$ into mice bearing transplanted, established Meth A tumors blocked LPS-induced tumor rejection (38). In addition, transplanted fibrosarcomas grew faster and more efficiently in mice treated with IFN- $\gamma$ specific mAbs. Moreover, IFN- $\gamma$ was shown to be involved in the antitumor effects of antibody-targeted superantigens (44). ELISpot is a sensitive functional assay used to measure INF- $\gamma$ production at the single cell level. The ELISpot showed there were much more tumor-specific INF- $\gamma$-producing cells in the mice bearing hepa1-6 hepatoma and B16 melanoma treated with Ad-MMRE-mTERT-BIS compared with those in other groups. $\mathrm{CD}^{+} \mathrm{CTLs}$ are one of the most crucial components among antitumor effectors (45). In our study, the results showed higher tumor-specific CTL activity was induced in the mice bearing hepa1-6 hepatoma and B16 melanoma treated with the recombinant adenoviruses compared with that in other groups. The ELISpot and cytotoxicity assays indicate Ad-MMRE-mTERT-BIS could induce stronger systemic antitumor immunity than either Ad-MMRE-mTERT-SEAtm or Ad-MMRE-mTERT-CD80. The survival period of the mice bearing hepa1-6 hepatoma or B16 melanoma treated with Ad-MMRE-mTERT-BIS was significantly longer and their tumors grew more slowly than those of mice in other groups. The regression of tumor indicates local antitumor immunity induced by Ad-MMRE-mTERT-BIS treatment.

In summary, our findings show that tumor cells infected with the recombinant adenovirus bearing SEA and CD80 genes can generate stronger antitumor immunity than the recombinant adenovirus bearing a single gene in vitro and 
in vivo, indicating that SEAtm and CD80 are able to stimulate antitumor immune responses synergistically. The same recombinant adenovirus bearing foreign gene controlled by TERT promoter may be used for targeting gene therapy of different kinds of tumors. The results provided experimental evidence that supports the feasibility and effectiveness of this novel approach in cancer immunotherapy. Additional underlying mechanisms need to be further studied.

\section{Acknowledgements}

This study was supported by the National Natural Sciences Foundation of China (no. 30772524).

\section{References}

1. Kotb M: Bacterial pyrogenic exotoxins as superantigens. Clin Microbiol Rev 8: 411-426, 1995.

2. Akbari A, Farahnejad Z, Akhtari J, Abastabar M, Mobini GR and Mehbod AS: Staphylococcus aureus enterotoxin B downregulates the expression of transforming growth factor-beta (TGF- $\beta$ ) signaling transducers in human glioblastoma. Jundishapur J Microbiol 9: e27297, 2016.

3. Akbari A, Mobini GR, Maghsoudi R, Akhtari J, Faghihloo E and Farahnejad Z: Modulation of transforming growth factor- $\beta$ signaling transducers in colon adenocarcinoma cells induced by staphylococcal enterotoxin B. Mol Med Rep 13: 909-914, 2016.

4. Zhao W, Li Y, Liu W, Ding D, Xu Y, Pan L and Chen S: Transcytosis, antitumor activity and toxicity of staphylococcal enterotoxin $\mathrm{C} 2$ as an oral administration protein drug. Toxins (Basel) 8: E185, 2016.

5. Zhang G, Xu M, Song Y, Su Z, Zhang H and Zhang C: TNF- $\alpha$ produced by SEC2 mutant (SAM-3)-activated human T cells induces apoptosis of HepG2 cells. Appl Microbiol Biotechnol 100: 2677-2684, 2016.

6. Mahmoodzadeh Hosseini H, Soleimanirad J, Mehdizadeh Aghdam E, Amin M and Imani Fooladi AA: Texosome-anchored superantigen triggers apoptosis in original ovarian cancer cells. Med Oncol 32: 409, 2015.

7. Terman DS, Serier A, Dauwalder O, Badiou C, Dutour A, Thomas D, Brun V, Bienvenu J, Etienne J, Vandenesch F, et al: Staphylococcal entertotoxins of the enterotoxin gene cluster (egcSEs) induce nitrous oxide- and cytokine dependent tumor cell apoptosis in a broad panel of human tumor cells. Front Cell Infect Microbiol 3: 38, 2013.

8. Kato M, Nakamura Y, Suda T, Ozawa Y, Inui N, Seo N, Nagata T, Koide Y, Kalinski P, Nakamura H, et al: Enhanced anti-tumor immunity by superantigen-pulsed dendritic cells. Cancer Immunol Immunother 60: 1029-1038, 2011.

9. Gong Z, Han C, Hao L, Yang J, Tang W and Teng G: Preparation and in-vitro bioactivity of a novel superantigen conjugate targeting bladder carcinoma. J Pharm Pharmacol 61: 869-875, 2009.

10. Dohlsten M, Hedlund G, Akerblom E, Lando PA and Kalland T: Monoclonal antibody-targeted superantigens: A different class of anti-tumor agents. Proc Natl Acad Sci USA 88: 9287-9291, 1991.

11. Litton MJ, Dohlsten M, Lando PA, Kalland T, Ohlsson L, Andersson J and Andersson U: Antibody-targeted superantigen therapy induces tumor-infiltrating lymphocytes, excessive cytokine production, and apoptosis in human colon carcinoma. Eur J Immunol 26: 1-9, 1996.

12. Gidlöf C, Dohlsten M, Lando P, Kalland T, Sundström C and Tötterman TH: A superantigen-antibody fusion protein for T-cell immunotherapy of human B-lineage malignancies. Blood 89: 2089-2097, 1997.

13. Ueno A, Arakawa F, Abe H, Matsumoto H, Kudo T, Asano R, Tsumoto K, Kumagai I, Kuroki M and Kuroki M: T-cell immunotherapy for human MK-1-expressing tumors using a fusion protein of the superantigen SEA and anti-MK-1 scFv antibody. Anticancer Res 22: 769-776, 2002.

14. Wahlsten JL, Mills CD and Ramakrishnan S: Antitumor response elicited by a superantigen-transmembrane sequence fusion protein anchored onto tumor cells. J Immunol 161: 6761-6767, 1998.
15. Si S, Sun Y, Li Z, Ge W, Zhang X, Hu P, Huang Y, Chen G, Song H, Huang Y, et al: Gene therapy by membrane-expressed superantigen for alpha-fetoprotein-producing hepatocellular carcinoma. Gene Ther 13: 1603-1610, 2006.

16. Lando PA, Dohlsten M, Hedlund G, Brodin T, Sansom D and Kalland T: Co-stimulation with B7 and targeted superantigen is required for MHC class II-independent T-cell proliferation but not cytotoxicity. Immunology 80: 236-241, 1993.

17. Si SY, Hu PZ, Huang YY, Ye J, Huang Y, Li ZS, Ge W, Li X, Qu P, Zhang XM, et al: Tumor cells with B7.1 and transmembrane anchored staphylococcal enterotoxin A generate effective antitumor immunity. Biochem Biophys Res Commun 347: 208-214, 2006.

18. Wirth T, Kühnel F and Kubicka S: Telomerase-dependent gene therapy. Curr Mol Med 5: 243-251, 2005.

19. Autexier C and Greider CW: Telomerase and cancer: Revisiting the telomere hypothesis. Trends Biochem Sci 21: 387-391, 1996.

20. Blackwood EM and Eisenman RN: Max: A helix-loop-helix zipper protein that forms a sequence-specific DNA-binding complex with Myc. Science 251: 1211-1217, 1991.

21. Kumagai T, Tanio Y, Osaki T, Hosoe S, Tachibana I, Ueno K, Kijima T, Horai T and Kishimoto T: Eradication of Myc-overexpressing small cell lung cancer cells transfected with herpes simplex virus thymidine kinase gene containing Myc-Max response elements. Cancer Res 56: 354-358, 1996.

22. Song JS: Adenovirus-mediated suicide SCLC gene therapy using the increased activity of the hTERT promoter by the MMRE and SV40 enhancer. Biosci Biotechnol Biochem 69: 56-62, 2005.

23. Si SY, Song SJ, Xu BX, Zhao G, Tan XQ, Liu JL, Zhang JZ and Liu ZG: Construction of recombinant adenovirus of SEA and CD80 genes co-expression regulated by mouse TERT promoter and identification of its expression in hepatoma cells. Xi Bao Yu Fen Zi Mian Yi Xue Za Zhi 27: 717-720, 2011 (In Chinese).

24. Nielsen SE, Zeuthen J, Lund B, Persson B, Alenfall J and Hansen HH: Phase I study of single, escalating doses of a superantigen-antibody fusion protein (PNU-214565) in patients with advanced colorectal or pancreatic carcinoma. J Immunother 23: $146-153,2000$

25. Cheng JD, Babb JS, Langer C, Aamdal S, Robert F, Engelhardt LR, Fernberg O, Schiller J, Forsberg G, Alpaugh RK, et al: Individualized patient dosing in phase I clinical trials: The role of escalation with overdose control in PNU-214936. J Clin Oncol 22: 602-609, 2004.

26. Ihle J, Holzer U, Krull F, Dohlsten M, Kalland T, Niethammer D and Dannecker GE: Antibody-targeted superantigens induce lysis of major histocompatibility complex class II-negative T-cell leukemia lines. Cancer Res 55: 623-628, 1995.

27. Holzer U, Bethge W, Krull F, Ihle J,Handgretinger R, Reisfeld RA, Dohlsten M, Kalland T, Niethammer D and Dannecker GE: Superantigen-staphylococcal-enterotoxin-A-dependent and antibody-targeted lysis of GD2-positive neuroblastoma cells. Cancer Immunol Immunother 41: 129-136, 1995.

28. Ma W, Yu H, Wang Q, Jin H, Solheim J and Labhasetwar V: A novel approach for cancer immunotherapy: Tumor cells with anchored superantigen SEA generate effective antitumor immunity. J Clin Immunol 24: 294-301, 2004.

29. Green JM, Turka LA, June CH and Thompson CB: CD28 and staphylococcal enterotoxins synergize to induce MHC-independent T-cell proliferation. Cell Immunol 145: 11-20, 1992.

30. Taub DD and Rogers TJ: Direct activation of murine T cells by staphylococcal enterotoxins. Cell Immunol 140: 267-281, 1992.

31. Lu SY, Sui YF, Li ZS, Pan CE, Ye J and Wang WY: Construction of a regulable gene therapy vector targeting for hepatocellular carcinoma. World J Gastroenterol 9: 688-691, 2003.

32. Pericuesta E, Ramírez MA, Villa-Diaz A, Relaño-Gines A, Torres JM, Nieto M, Pintado B and Gutiérrez-Adán A: The proximal promoter region of mTert is sufficient to regulate telomerase activity in ES cells and transgenic animals. Reprod Biol Endocrinol 4: 5-16, 2006.

33. Si SY, Song SJ, Zhang JZ, Liu JL, Liang S, Feng K, Zhao G and Tan XQ: Cloning of mouse telomerase reverse transcriptase gene promoter and identification of proximal core promoter sequences essential for the expression of transgenes in cancer cells. Oncol Rep 26: 377-382, 2011.

34. Tian XL, Yan Z, Chen J, Zhao WH and Guo W: Clinical application of highly agglutinative staphylococcin in cancer treatment updates of the literature. Eur Rev Med Pharmacol Sci 20: 2718-2725, 2016. 
35. Dohlsten M, Sundstedt A, Björklund M, Hedlund G and Kalland T: Superantigen-induced cytokines suppress growth of human colon-carcinoma cells. Int J Cancer 54: 482-488, 1993.

36. Lando PA, Dohlsten M, Hedlund G, Akerblom E and Kalland T: $\mathrm{T}$ cell killing of human colon carcinomas by monoclonalantibody-targeted superantigens. Cancer Immunol Immunother 36: 223-228, 1993.

37. Lando PA, Hedlund G, Dohlsten M and Kalland T: Bacterial superantigens as anti-tumour agents: Induction of tumour cytotoxicity in human lymphocytes by staphylococcal enterotoxin A. Cancer Immunol Immunother 33: 231-237, 1991.

38. Dighe AS, Richards E, Old LJ and Schreiber RD: Enhanced in vivo growth and resistance to rejection of tumor cells expressing dominant negative IFN $-\gamma$ receptors. Immunity 1: 447-456, 1994

39. Kaplan DH, Shankaran V, Dighe AS, Stockert E, Aguet M, Old LJ and Schreiber RD: Demonstration of an interferon gamma-dependent tumor surveillance system in immunocompetent mice. Proc Natl Acad Sci USA 95: 7556-7561, 1998.

40. Shankaran V, Ikeda H, Bruce AT, White JM, Swanson PE, Old LJ and Schreiber RD: IFNgamma and lymphocytes prevent primary tumour development and shape tumour immunogenicity. Nature 410: 1107-1111, 2001.
41. Street SE, Cretney E and Smyth MJ: Perforin and interferongamma activities independently control tumor initiation, growth, and metastasis. Blood 97: 192-197, 2001.

42. Street SE, Trapani JA, MacGregor D and Smyth MJ: Suppression of lymphoma and epithelial malignancies effected by interferon gamma. J Exp Med 196: 129-134, 2002.

43. Nishimura T, Nakui M, Sato $M$, Iwakabe K, Kitamura $H$, Sekimoto M, Ohta A, Koda T and Nishimura S: The critical role of Th1-dominant immunity in tumor immunology. Cancer Chemother Pharmacol 46 (Suppl): S52-S61, 2000.

44. Rosendahl A, Kristensson K, Hansson J, Riesbeck K, Kalland T and Dohlsten M: Perforin and IFN-gamma are involved in the antitumor effects of antibody-targeted superantigens. J Immunol 160: 5309-5313, 1998.

45. Melief CJ and Kast WM: T-cell immunotherapy of tumors by adoptive transfer of cytotoxic $\mathrm{T}$ lymphocytes and by vaccination with minimal essential epitopes. Immunol Rev 145: 167-177, 1995. 